\title{
PARTICIPAÇÃO E CULTURA POLÍTICA: RENDIMENTOS SUBJETIVOS DA EXPERIÊNCIA DO ORÇAMENTO PARTICIPATIVO ${ }^{1}$
}

\author{
Ednaldo Aparecido Ribeiro
}

Julian Borba

\begin{abstract}
Resumo
O Orçamento Participativo tem sido apontado pela literatura como uma inovação democrática portadora de virtudes, seja no plano da sua capacidade de racionalização da ação estatal, seja no potencial de alterar padrões históricos de relacionamento Estado x Sociedade no Brasil, como o clientelismo. Estudos recentes também apontam para eventuais impactos da experiência nos padrões atitudinais e comportamentais dos participantes, de modo que o OP produziria algum tipo de aprendizado político. Procurando explorar no plano empírico esses potenciais efeitos positivos o presente trabalho se propõe a analisar em que medida o envolvimento nessa instituição participativa pode ser entendido como condicionante ou determinante de um conjunto de indicadores reunidos pela literatura convencional no rótulo de cultura política. Nesse sentido, pretendemos questionar se é possível, a partir de inovações institucionais participativas, produzir alterações no padrão de orientações subjetivas e atitudes dos cidadãos. Para alcançar esses objetivos tomamos como material empírico um conjunto de dados referentes à experiência do Orçamento Participativo desenvolvida em Porto Alegre. Tais informações são provenientes de survey conduzido pelo Núcleo de Pesquisas Sobre a América Latina (NUPESAL) no ano de 2000. O trabalho está organizado em três partes: na primeira, discutimos as questões teóricas envolvidas em tal debate. Na segunda, apresentamos os dados empíricos. Nas considerações finais, são estabelecidas algumas conclusões e perspectivas para novos trabalhos.
\end{abstract}

Palavras-chaves: Participação; Orçamento Participativo; Cultura Política.

\begin{abstract}
The Participatory Budget has been described in literature as a democratic innovation bearer of virtues, whether in the capacity for rationalization of state action, is the potential to alter historical patterns of relationship State $x$ Society in Brazil, such as clientelism. Recent studies also point to possible impacts of experience in attitudinal and behavioral patterns of participants, so the OP would produce some kind of political learning. Looking empirically explore these potential positive effects of this study is to examine to what extent the participatory involvement in this institution can be understood as conditioning or determining a set of indicators collected by the conventional literature on the label of political culture. Therefore, we intend to question whether it is possible, from participatory institutional innovations, produce

\footnotetext{
${ }^{1} \mathrm{O}$ presente paper é um desdobramento de estudo anterior, onde abordamos exclusivamente os impactos do OP nos tradicionais indicadores de capital social. A atual versão amplia o enfoque para um conjunto maior de variáveis tradicionalmente utilizadas nos estudos de socialização e cultura política.
} 
changes in the pattern of subjective orientations and attitudes of citizens. To achieve these goals we take as a set of empirical data on the experience of participatory budgeting in Porto Alegre developed. Such information comes from one survey conducted by the Center for Research on Latin America (NUPESAL) for the year 2000. The paper is organized into three parts: First, we discuss the theoretical issues involved in this debate. On Monday, we present the empirical data. In the end, we set out some conclusions and perspectives for further work.

Keywords: Participation; Participatory Budgeting; Political Culture.

\section{Introdução}

O Orçamento Participativo (OP) tem sido apontado pela literatura como uma inovação democrática (AVRITZER e NAVARRO, 2003) portadora de virtudes, seja no plano da sua capacidade de racionalização da ação estatal (FEDOZZI, 1997), seja no potencial de alterar padrões históricos de relacionamento Estado x Sociedade no Brasil, como o clientelismo (ABERS, 1998). Estudos recentes também apontam para eventuais impactos da experiência nos padrões atitudinais e comportamentais dos participantes, de modo que o OP produziria algum tipo de aprendizado político (FEDOZZI, 2002; LÜCHMANN, 2008).

O presente trabalho pretende apresentar resultados de testes empíricos sobre esse suposto efeito pedagógico do envolvimento dos cidadãos nessa inovação institucional. Buscamos verificar se a participação no OP pode ser um preditor relevante para um conjunto de medidas relacionadas à cultura política dos indivíduos, especialmente ligadas ao tema do interesse e do ativismo político. A ideia é confrontar tais indicadores com outras variáveis geralmente presentes na literatura através da construção de modelos multivariados que permitem avaliar comparativamente impactos produzidos por distintas medidas independentes.

As razões para tal esforço de investigação são de ordens empírica e teórica: em termos empíricos pela inexistência de consenso quanto a real capacidade do OP em promover mudança de valores e comportamentos. Neste sentido, o trabalho poderia oferecer evidências empíricas para iluminar essa discussão. Com relação à questão teórica, o exame de alguns indicadores relacionados às dimensões dos valores e atitudes políticas nos possibilita testar (e eventualmente questionar), a hipótese que vê a socialização primária como o momento fundante da cultura política dos indivíduos. Como o envolvimento com a inovação institucional, aqui focalizada, ocorre durante a vida adulta do cidadão, a investigação sobre seus efeitos pode oferecer informações relevantes sobre o papel das experiências políticas que ocorrem na vida adulta para os processos de formação e alteração dos valores, crenças e atitudes.

A base empírica utilizada no trabalho se refere à experiência do Orçamento Participativo desenvolvida em Porto Alegre e foi construída por um 
survey sobre comportamento político e eleições (NUPESAL, 2000), aplicado à amostra representativa da população municipal $(n=533)^{2}$.

O trabalho está organizado em quatro partes: na primeira, discutimos as questões teóricas envolvidas em tal debate. Aí também fazemos uma apresentação da literatura que estudou o OP de Porto Alegre sob a ótica da cultura política. Na segunda, apresentamos a metodologia utilizada na análise dos dados para, na parte três, discutir um conjunto de resultados de testes bivariados e multivariados. Nas considerações finais, são estabelecidas algumas conclusões e perspectivas para novos trabalhos.

\section{Cultura Política e Socialização}

Ainda que a relevância de fatores culturais na explicação de fenômenos políticos permaneça objeto de considerável polêmica entre os cientistas sociais, dificilmente podemos encontrar investigadores que ostensivamente afirmem a total irrelevância dessa dimensão subjetiva. Apesar do tema da causalidade e da capacidade explicativa de variáveis relacionadas à cultura política ser controverso (MULLER e SELIGSON, 1994), a tese de que tais fatores importam tem cada vez mais ganhado força (PUTNAM, 1997; NORRIS, 2002; INGLEHART e WELZEL, 2009). Mesmo entre pesquisadores não vinculados ao que podemos chamar de abordagem culturalista têm se difundido a tese de que o conjunto de fatores relacionados a essa dimensão subjetiva deve ser considerado em qualquer análise multicausal dos problemas contemporâneos, ainda que na posição de variável interveniente (DAHL, 1997).

Esse posicionamento se mostra ainda mais defensável quando os objetos de estudos são as chamadas novas democracias da América Latina, estabelecidas no que Huntington (1991) denominou de terceira onda de democratização. Apontando os limites das abordagens schumpeterianas sobre a transição e a consolidação da democracia nesta região, autores apontam que a manutenção do procedimento institucionalizado de alternância das elites não deve ser a única preocupação das análises sobre a situação contemporânea da democracia latino-americana (VITULO, 2001; MONCLAIRE, 2001; VIGEVANI e OLIVEIRA, 2005).

Como sua proposta de superação dos limites estritamente institucionais a abordagem culturalista tem ganhado bastante destaque nas últimas décadas e tem tornado comum o emprego de termos como capital social, confiança interpessoal, cultura cívica e cultura política, tanto dentro quanto fora da academia.

É preciso reconhecer que a preocupação com tais determinantes culturais não é recente, sendo possível identificar seus antecedentes em escritos políticos de filósofos da antiguidade. Sua sistematização como programa de pesquisas, porém, é algo recente, datando da segunda metade

2 Para maiores detalhes sobre plano amostral, ver NUPESAL (2000). 
do século XX. Como marco desta institucionalização, temos o estudo pioneiro de Gabriel Almond e Sidney Verba, The Civic Culture (1989), inspirador de uma série de novas pesquisas que trataram de aprofundar esta temática que atualmente vive um período de intensa produção.

Partindo do pressuposto de que os valores, sentimentos, crenças e conhecimentos são relevantes para explicar os padrões de comportamentos políticos adotados pelos indivíduos, esses dois pesquisadores desenvolveram um estudo que se estendeu por cinco países (Estados Unidos, Inglaterra, Itália, Alemanha e México), pretendendo investigar o grau de congruência entre esse conjunto de variáveis subjetivas e o sistema político.

O conceito de cultura política, orientador de toda a investigação, foi definido originalmente como "[...] the particular distribution of patterns of orientation toward political objects among the members of the nation [...]", ou seja, um conjunto de orientações políticas subjetivas que poderiam explicar a motivação subjacente às ações praticadas pelos atores, neste caso específico, tendo como referência os objetos políticos (ALMOND e VERBA, 1989, p. 13).

Como todo empreendimento inovador tal formulação inicial não foi poupada de críticas. Alguns autores inicialmente trataram de questionar o demasiado etnocentrismo presente na definição do que deveria ser considerado como político (INGLEHART, 1988). De fato, Almond e Verba tomam o modelo anglo-saxão de democracia liberal como o que teria as condições necessárias para o surgimento do modelo de cultura cívica. Em decorrência, ao definirem as características subjetivas de cada um dos modelos de cultura política os autores teriam, por um lado, colocado em posição confortável países como Estados Unidos e Inglaterra e, por outro, condenado países como Alemanha e Itália a uma situação política desfavorável (RENNó, 1998).

O problema estaria na adoção de uma determinada perspectiva teórica que restringe o conceito de política às instituições formais de caráter representativo, reduzindo o campo de visão aos limites das representações culturais compatíveis com a ideologia dominante. Dessa forma, adotando o paradigma minimalista de democracia, toda e qualquer manifestação de valores contrários às regras do jogo político liberal é interpretada como incongruente com a democracia em sua totalidade. Tomando o político como um dado inquestionável, fecha os olhos para o fato de que existem diferentes concepções sobre o que seria ou não a dimensão política da ação humana em conflito (ALVAREZ, DAGNINO e ESCOBAR, 2000).

Após um momento de reflexão crítica e rompendo com estes limites iniciais ${ }^{3}$, os estudos sobre essa dimensão cultural da política atualmente

\footnotetext{
3 O artigo de Inglehart (1988), intitulado The renaissance of political culture, é, por muitos autores, considerado o momento paradigmático da renovação do enfoque da cultura política.
} 
passam por um momento de intensa produção. Tal retomada pode ser atribuída em grande medida à identificação de deficiências explicativas nas abordagens estritamente institucionais, especialmente na análise das novas democracias (BAQUERO, 2003). Este renascimento da abordagem culturalista tem sido marcado pela diversidade de propostas, merecendo destaque as formuladas por Robert Putnam (1997), Robert Dahl (1997), Inglehart (2001) e Inglehart e Welzel (2005).

Dentro dessa renovada agenda de pesquisas em cultura política enfocamos nesse artigo especificamente uma questão que pode ser considerada central para a compreensão das relações entre cultura e instituições: como são formados (ou alterados) os valores, crenças e atitudes que compõem a cultura política?

Essa pergunta pode ser considerada ainda mais relevante no contexto das chamadas novas democracias e regimes democráticos em processo de consolidação. No Brasil vários pesquisadores têm se dedicado a investigações sobre o caráter da cultura política nacional e suas principais conclusões têm apontado para a permanência de um padrão de comportamento político orientado por crenças que dificultariam a implementação de relações políticas pautadas pela horizontalidade e pela igualdade, portanto, constituindo-se como obstáculo ao estabelecimento de uma legitimidade democrática em nosso país (O'DONNELL, 1988; AVRITZER, 1995; CARVALHO, 1999; CHAUÍ, 2000; BAQUERO, 2002).

A explicação para a permanência deste padrão passa necessariamente pelo desvendamento dos processos de aquisição, incorporação, alteração e substituição dos elementos que compõem a cultura política dos cidadãos. O estudo do papel das instituições responsáveis pela transmissão dos valores, crenças e conhecimentos às novas gerações é dessa forma fundamental, não obstante, é preciso considerar também o conjunto de experiências que ocorrem na vida pré-adulta e adulta dos indivíduos como potencialmente relevantes nesses processos. A despeito de sua importância, os pesquisadores ainda de maneira muito incipiente têm-se dedicado à temática da socialização política, em especial no que diz respeito aos estudos nacionais, o que tem dificultado a compreensão sobre o processo de formação de atitudes políticas (SCHMIDT, 2001).

Como afirmam Almond e Verba, o processo deformação do padrão de atitudes e comportamentos de adultos em relação aos objetos políticos envolve um complexo de fatores. Questionando abordagens psicológicas que afirmavam que a formação deste padrão se daria apenas nos primeiros anos da vida dos indivíduos, os autores argumentam que experiências na chamada fase pré-adulta e também na maturidade têm importância fundamental para a configuração das orientações políticas subjetivas. Isso não quer dizer que as experiências da primeira infância não tenham relevância, mas sim que "early socialization experiences significantly affect individual's basic personality predispositions and may therefore affect his political behavior, but numerous other factors intervene between these earliest experiences and later political 
behavior that greatly inhibit the impact of the former on the latter" (ALMOND e VERBA, 1989, p. 266-267).

Começando na infância, mas se estendendo por todas as demais fases da vida do indivíduo, devemos definir o processo de socialização política como "[...] o conjunto de experiências que, no processo de formação da identidade social do indivíduo, tem influência na cristalização do seu papel como ator significativo ou não no sistema político e as crenças que ele desenvolve em relação à política e as instituições [...]" (BAQUERO, 1997). Sendo assim, dáse continuamente, na medida em que as pessoas vivenciam suas experiências sociais em espaços distintos como a família e a escola; os seus padrões de atitudes e procedimentos vão sendo formados e/ou alterados (ALMOND e POWELL, 1972).

Essa hipótese do desenvolvimento contínuo, entretanto, não impede o questionamento sobre os diferentes pesos que os distintos momentos de aprendizado produzem sobre a cultura política. Robert Dahl (1997), respondendo a essa questão, afirma a importância da socialização precoce diante dos demais momentos e experiências políticas. Para esse autor, as crenças acerca dos objetos políticos são adquiridas nas primeiras duas décadas de vida de uma pessoa, período em que se é mais receptivo a elas. Quando se ultrapassa esse período os indivíduos tendem a possuir uma visão mais cristalizada e suas crenças tendem a se tornarem mais estáveis e menos suscetíveis a mudanças (DAHL, 1997, p. 160).

A refutação dessa afirmação envolve sérios desafios metodológicos que só podem ser parcialmente satisfeitos pela condução de estudos de painel com a maior duração possível. Tomando cidadãos adultos como público, sondagens sucessivas com intervalos regulares deveriam ser conduzidas para aferir de fato se ocorrem alterações nas orientações subjetivas individuais na medida em que os cidadãos tomam parte de experiências no interior dos mais variados espaços, tais como o ambiente de trabalho, o sindicato, o clube esportivo, a associação de moradores, o conselho de saúde, o orçamento participativo. Apenas com esse tipo de acompanhamento complexo e operacionalmente dispendioso uma resposta satisfatória pode ser dada à questão da relevância das experiências de socialização adulta.

Infelizmente os dados oriundos de pesquisas de painéis ainda são raros, o que nos obriga a buscar alternativas metodológicas para procurar responder inquietações dessa ordem. Na seção metodológica apresentamos os procedimentos adotados para tentar contornar essas limitações empíricas. Antes disso, na próxima seção apresentamos brevemente uma síntese sobre a experiência do Orçamento Participativo na cidade de Porto Alegre.

\section{OP e Cultura Política em Porto Alegre}

Como já destacamos, o OP vem se constituindo numa importante inovação na gestão pública brasileira, pelo fato de potencialmente romper com as práticas tradicionais, exercidas através de relações convencionalmente 
denominadas de clientelistas e patrimonialistas. Tal rompimento se daria pelo caráter universalista e racionalizador existente nas suas regras de funcionamento (FEDOZZI, 1997 e 2002), as quais seriam aplicadas através do processo de participação dos cidadãos, quando chamados a participar do processo decisório estatal.

Não é nossa intenção nesse artigo discutir os diferentes modelos de OP, nem apresentar como se dá a participação dos indivíduos no processo, pois isso já foi feito por outros autores (AVRITZER e NAVARRO, 2003; ABERS, 1998; LÜCHMANN, 2002; PONTUAL, 2001; BORBA e LÜCHMANN, 2007).

Como já amplamente destacado pela literatura, a difusão do OP ocorreu a partir do modelo da cidade de Porto Alegre (desde 1989) a partir de uma tentativa de articulação entre os princípios da democracia representativa e da democracia participativa. De acordo com as características institucionais da democracia brasileira, o Poder Executivo possui a prerrogativa de iniciar e coordenar todo o processo de definição acerca dos tributos, das finanças e do orçamento público. No âmbito municipal, o Executivo é responsável pela elaboração do projeto de lei orçamentária anual, devendo enviá-lo ao Poder Legislativo que possui, entre outras atribuições, a prerrogativa de apreciar, apresentar emendas, aprovar a peça orçamentária e fiscalizar a sua execução. A participação da população em todo este processo limita-se, via de regra, à escolha dos representantes dos poderes executivo e legislativo por meio do sufrágio universal ${ }^{4}$.

Com a instituição do OP foram estabelecidos mecanismos de participação direta e representativa nesse processo de elaboração e execução orçamentária. Em Porto Alegre a experiência do OP teve início com a vitória do PT nas eleições municipais de $1988^{5}$ e sua arquitetura institucional é composta por três instâncias: 1) executivo municipal, responsável pelo gerenciamento e processamento técnico-político da discussão com a população; 2) instâncias comunitárias autônomas, formadas principalmente por organizações da sociedade civil de base regional que articulam a participação dos moradores e a escolha das prioridades das regiões (conselhos populares, associações de moradores, união de vilas, etc.); e 3) instâncias institucionais de participação e representação popular, com funções de co-gestão dos recursos públicos (Conselho do Orçamento Participativo COP, Assembléias Regionais, Fórum Regional do Orçamento, Plenárias Temáticas e Fórum Temático do Orçamento) (LÜCHMANN e BORBA, 2010).

\footnotetext{
4 Deve-se destacar que a população pode também realizar demandas diretas aos poderes constituídos. A prática mais usual no Brasil de inclusão de demandas no orçamento público são as emendas parlamentares.

5 O PT administrou Porto Alegre até 2004, quando foi derrotado pelo candidato José Fogaça (na época, ligado ao Partido Popular Socialista). Com a mudança de governo, a estrutura do OP sofreu várias alterações, o que tem provocado uma intensa discussão nos meios políticos e acadêmicos. Uma análise sistemática das mudanças implementadas pelo novo governo e suas críticas pode encontrada no site da ONG CIDADE (http://www.cidade.org.br).
} 
Tal estrutura opera através de assembleias regionais e temáticas em que se escolhem, através de notas, as prioridades de cada região. Estas notas são somadas às notas dos outros dois critérios gerais (carência do serviço ou infra-estrutura e população total da região). Num segundo momento são eleitos conselheiros do COP: dois titulares e dois suplentes de cada região e temática. O COP discute e aprova o montante total de recursos do orçamento, acompanha a execução das obras aprovadas e discute os critérios técnicos apresentados pelo governo.

Embora com diversas variações, esse modelo foi sendo paulatinamente implementado em diferentes regiões do país, e contabilizou, no período de 2005 a 2008, 201 experiências (AVRITZER e WAMPLER, 2008). Vários trabalhos têm apontado que tal difusão do Orçamento Participativo tem produzido também uma proliferação de distintos modelos institucionais que se autodenominam $\mathrm{OP}^{6}$.

Os impactos objetivos dessa inovação institucional sob a governança democrática nos locais em que foram implementadas tem sido objeto de extensa bibliografia e continua despertando a atenção dos pesquisadores. Os seus rendimentos subjetivos, entretanto, tem sido discutidos de forma um tanto quanto intuitiva pela literatura, em especial devido aos problemas metodológicos envolvidos nesse tipo de investigação, como já mencionamos anteriormente. Estudos tomando como público apenas os participantes do OP têm atestado os efeitos positivos dessa experiência em termos de mudança na cultura política e/ou aprendizado (FEDOZZI, 2002; LÜCHMANN, 2008).

Outras investigações, entretanto, ao trabalharem com amostras do público massivo apontam para interpretações divergentes. De um lado, Baquero et al (2005, p. 123), ao testar as relações entre participação no OP e o desenvolvimento de novas atitudes para com o Estado (em particular sobre os impostos), avaliam que:

\begin{abstract}
[...] a situação social e econômica da população exerce influência superior ao OP na estruturação de atitudes e comportamentos em relação aos impostos. Tudo indica que a falta de legitimidade dos impostos, mais do que uma questão de competência cultural, é um problema de solução pragmática.
\end{abstract}

Já Paulo Krishke (2004 e 2005), ao comparar a cultura política de porto-alegrenses e curitibanos, identifica na primeira cidade uma série de predisposições atitudinais, no sentido de valorização da política e da participação da população em projetos coletivos. Nesse sentido, seguindo a perspectiva do autor, os vários anos de administração petista e as políticas de

\footnotetext{
${ }^{6}$ Ver em especial Avritzer e Navarro (2003); Borba e Lüchmann (2007).
} 
OP teriam desempenhado um papel significativo na conformação dessa cultura política.

Considerando essas interpretações discordantes sobre os efeitos atitudinais e valorativos do OP, o presente trabalho se propõe a testar empiricamente o impacto dessas experiências adultas sobre um conjunto de indicadores relacionados à cultura política.

\section{Procedimentos metodológicos}

Em razão da indisponibilidade de dados de painel aplicados aos participantes do OP, somos obrigados a buscar alternativas que possam produzir respostas satisfatórias para o problema proposto. Essa solução deve necessariamente oferecer condições para aferir em que medida a experiência de participar no OP impacta orientações políticas subjetivas dos indivíduos. Acreditamos que a base de dados produzida pela pesquisa Comportamento Político e Eleições (NUPESAL, 2000) oferece condições para a realização do teste, pois fornece informação não apenas sobre o envolvimento dos indivíduos nessa instituição, mas também sobre a duração dessa experiência.

Nossa variável independente principal, portanto, é o tempo de participação no OP (TOP), codificada na seguinte escala: $0=$ nunca participou, $1=$ há menos de um ano, $2=$ dois anos, $3=$ três anos e $4=$ sempre participou. As variáveis de controle dos efeitos são escolaridade, renda, idade e sexo. A decisão de incluir escolaridade e renda está relacionada ao fato de que ambas são geralmente apontadas por parte da literatura como as de maior capacidade de predição quanto a diferentes indicadores de cultura política. Sexo e idade, ainda que não apareçam com a mesma relevância nos estudos sobre o tema, foram incluídas por se tratarem de importantes atributos demográficos.

No campo das variáveis dependentes, procuramos selecionar aquelas que representassem temas relevantes para a abordagem culturalista. É preciso alertar, todavia, que a base de dados utilizada é produto de uma pesquisa sobre comportamento eleitoral, logo, temas caros aos estudos sobre cultura política estão ausentes.

A primeira variável destacada é uma medida do interesse dos entrevistados por política (INTEPOL), empregado com frequência nos estudos da área como indicador de uma postura participativa favorável à democracia (ALMOND e VERBA, 1989; INGLEHART, 2001). Em nosso caso trata-se de um escala de interesse codificada como segue: $0=$ nem um pouco, $1=$ muito pouco, $2=$ mais ou menos, $3=$ muito.

O segundo indicador selecionado diz respeito à disposição dos entrevistados para votar levando em consideração a situação hipotética do fim da obrigatoriedade (VOTO). Trata-se, portanto, de uma medida relacionada à anterior, pois reflete igualmente uma postura politicamente interessada. Sua codificação é binária, sendo $0=$ não e $1=\operatorname{sim}$. 
Ainda sobre esse tema do interesse específico por meio do voto, adicionamos também a variável obtida através da seguinte pergunta: $O(A)$ sr.(a) costuma acompanhar as atividades dos candidatos após a eleição? (ACCAND) Neste caso a codificação original possibilita três respostas: $0=$ não, $1=$ às vezes e $2=\operatorname{sim}$.

Ainda no que diz respeito aos processos eleitorais, construímos um Índice de Ativismo Eleitoral (IAE), que serve como uma medida do quanto o interesse manifesto pelos entrevistados se converte em um comportamento engajado. Esse índice foi elaborado de forma somatória a partir dos seguintes itens: participação em passeatas e carreatas de candidatos, doação de dinheiro para campanha, trabalho gratuito para um candidato, colocação de cartazes em casa ou no local de trabalho, comparecimento em debates e reuniões, audiência do horário eleitoral na TV e também no Rádio. São ao todo sete variáveis, todas codificadas com três valores: $0=$ não, $1=$ às vezes e $2=\operatorname{sim}$. Como resultado temos um índice que varia de 0 a 14 . 0 teste Alpha de Cronbach retornou o valor de 0.83 , o que indica considerável consistência interna para essa medida integrada de ativismo.

Para finalizar, para além da participação eleitoral também incluímos uma variável sobre a participação dos entrevistados em grupos ou associações em geral (ASSOC), uma medida binária ( $0=$ não e $1=\operatorname{sim}$ ).

Definidas as variáveis, procedemos às análises em duas etapas distintas. Na primeira foram conduzidos testes bivariados de associação. $\mathrm{Na}$ sequência, para confirmar a aprofundar a compreensão sobre as associações encontradas, foram construídos modelos multivariados de regressão linear e logística, dependendo da natureza das variáveis dependentes.

O modelo estatístico-matemático simples de regressão relaciona uma variável $Y$, denominada variável resposta ou dependente, com uma segunda medida $X$, chamada de variável explicativa, independente ou preditora. Como no caso dos testes de correlação e associação, esse tipo de análise toma as observações singulares como pares de dados $(x, y)$ relativos às variáveis envolvidas na equação. Quando um determinado valor de $y$ depende parcialmente do valor do seu correspondente $x$ podemos falar de uma relação linear entre essas variáveis. Como trabalhamos com mais de uma variável explicativa nas análises apresentadas ao longo desse trabalho, nos valemos principalmente de modelo de regressão múltipla. Levando em consideração que uma variável dependente geralmente varia em relação a mais de uma medida preditora, esse tipo de procedimento nos permite conhecer a influência de cada uma dessas sobre $y$. Esse procedimento possibilita testar se o relacionamento verificado entre as variáveis envolvidas é realmente válido ou se é espúrio, sobretudo porque podemos controlar os efeitos da nossa variável independente principal com a inclusão de terceiras variáveis nos modelos. Destacamos desde já que nossa intenção com a aplicação dessa técnica estatística não é construir modelos explicativos robustos acerca das variáveis respostas, mas tão somente identificar a intensidade e a 
consistência do efeito produzido pela participação no Orçamento Participativo sobre cada uma das variáveis dependentes mencionadas anteriormente.

Em alguns casos, entretanto, a natureza das variáveis dependentes não possibilita o emprego dessa técnica, que pressupõe a natureza quantitativa das medidas. Nos momentos em que trabalhamos com variáveis dicotômicas, como no caso da confiança interpessoal, empregamos modelos de regressão logística, especialmente desenvolvidos para esse nível de mensuração (POWERS e XIE, 2008).

\section{Resultados}

Começando pelos testes bivariados verificamos associações estatisticamente significativas em todos os cruzamentos. Na Tabela 1 podemos ver claro crescimento no grupo dos que se interessam muito na medida em que avançamos na escala que mede o tempo de envolvimento no OP. Movimento inverso ocorre na coluna da categoria nem um pouco. Nas colunas intermediárias essa relação não deixa de ser linear, mas ainda assim constatamos um coeficiente de associação estatisticamente significativo $(p=0.000)$ com intensidade de fraca à moderada $(y=0.27)$. Neste caso, podemos afirmar a existência de relação positiva entre o envolvimento com essa inovação institucional e a ocorrência de uma postura politicamente interessada. A consistência desse efeito será testada à frente com a inclusão de variáveis de controle em modelos multivariados.

Tabela 1 - Associação entre Tempo de OP e Interesse por Política

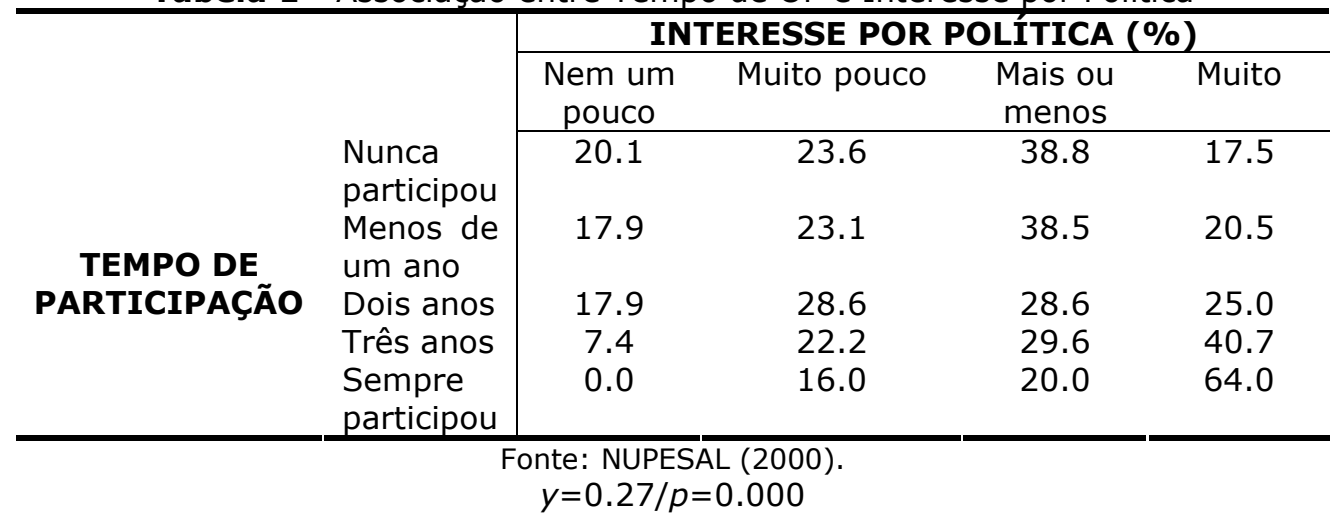

Associação com intensidade um pouco maior (0.34) é encontrada quando cruzamos o tempo de OP com a disposição para votar em um contexto hipotético de não obrigatoriedade (Tabela 2). As duas colunas da tabela possuem sentidos inversos na medida em que avançamos na escala de tempo da participação. A linearidade só é quebrada entre a categoria dos que participam a menos de um ano, que apresentam com maior intensidade a 
disposição para votar. Esse resultado reforça o anterior e indica que a experiência participativa está de forma consistente relacionada com disposições positivas em relação à participação política.

Tabela 2 - Associação entre Tempo de OP e Voto não obrigatório

\begin{tabular}{cl|cc}
\hline \multirow{2}{*}{ TEMPO DE } & VOTO NÃO OBRIGATÓRIO (\%) \\
\cline { 3 - 4 } PARTICIPAÇÃo & Não & Sim \\
\cline { 3 - 4 } & Menos de um ano & 33.8 & 66.2 \\
& Dois anos & 15.4 & 84.6 \\
& Três anos & 32.0 & 68.0 \\
& Sempre participou & 19.2 & 80.8 \\
& \multicolumn{2}{c}{ Fonte: NUPESAL $(2000)}$. \\
\hline \multicolumn{3}{c}{$y=0.34 / p=.000$}
\end{tabular}

Nível de associação muito próximo ao anterior (0.35), foi verificado no cruzamento com a variável sobre a acompanhamento das atividades dos candidatos após as eleições (Tabela 3). Novamente verificamos elevações, ainda que não totalmente lineares, na coluna das respostas positivas na medida em que a escala de tempo avança. Desta forma, além de estar relacionado com o interesse por política, o envolvimento no OP também parece estar ligado a uma postura fiscalizadora.

Tabela 3 - Associação entre Tempo de OP e Acompanhamento das Atividades do Candidato

\begin{tabular}{|c|c|c|c|}
\hline & 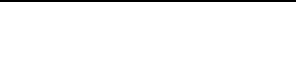 & \multicolumn{2}{|c|}{$\begin{array}{l}\text { ACOMPANHAMENTO DO } \\
\text { CANDIDATO }(\%)\end{array}$} \\
\hline & \multirow[b]{2}{*}{ Nunca participou } & Não & Sim \\
\hline & & 26.1 & 73.9 \\
\hline & $\begin{array}{l}\text { Menos de um } \\
\text { ano }\end{array}$ & 18.9 & 81.1 \\
\hline \multirow{3}{*}{$\begin{array}{c}\text { TEMPO DE } \\
\text { PARTICIPAÇÃO }\end{array}$} & Dois anos & 22.2 & 77.8 \\
\hline & Três anos & 3.8 & 96.2 \\
\hline & $\begin{array}{l}\text { Sempre } \\
\text { participou }\end{array}$ & 12.0 & 88.0 \\
\hline
\end{tabular}

No que diz respeito ao envolvimento dos entrevistados com o processo eleitoral, medido pelo Índice de Ativismo Eleitoral (IAE), a associação pode ser considerada de moderada à forte $(0.051)$, indicando que avanços no tempo de experiência no OP são acompanhados de acréscimo na escala de envolvimento nas atividades relacionadas a esse momento chave da vida política nacional. Esse dado vem, portanto, corroborar a hipótese do impacto 
positivo do envolvimento nessa instituição sobre as orientações e atitudes políticas individuais.

Tabela 4 - Associação entre Tempo de OP e Participação Eleitoral

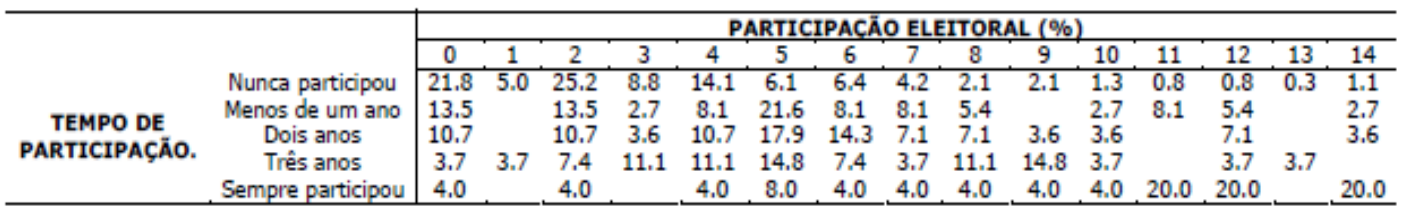

Fonte: NUPESAL (2000).

$$
y=0.51 / p=0.000
$$

Situação semelhante pode ser verificada quando nossa atenção se desloca dessa dimensão da política institucional em direção à participação dos cidadãos em grupos e associações. A intensidade da associação verificada nesse caso também é moderada (0.49) e revela a existência de relacionamento positivo entre o tempo de envolvimento com o OP e o engajamento nesse tipo de instituição da sociedade civil.

Tabela 5 - Associação entre Tempo de OP e Participação em Associações

\begin{tabular}{|c|c|c|c|}
\hline \multirow{7}{*}{$\begin{array}{c}\text { TEMPO DE } \\
\text { PARTICIPAÇÃO }\end{array}$} & \multirow{7}{*}{$\begin{array}{l}\text { Nunca participou } \\
\text { Menos de um ano } \\
\text { Dois anos } \\
\text { Três anos } \\
\text { Sempre } \\
\text { participou }\end{array}$} & \multicolumn{2}{|c|}{$\begin{array}{l}\text { PARTICIPAÇÃO EM } \\
\text { ASSOCIAÇÕES (\%) }\end{array}$} \\
\hline & & Não & Sim \\
\hline & & 82.1 & 17.9 \\
\hline & & 71.1 & 28.9 \\
\hline & & 61.5 & 38.5 \\
\hline & & 48.1 & 51.9 \\
\hline & & 52.0 & 48.0 \\
\hline
\end{tabular}

Para confirmar tais resultados, e assim evitar possíveis conclusões espúrias, conduzimos adicionalmente análises multivariadas que incluem no grupo de variáveis independentes alguns atributos sociais e demográficos fundamentais. Nossa intenção é verificar se o efeito da participação no OP continua relevante na presença dessas características que potencialmente podem afetar as variáveis dependentes analisadas acima.

Em se tratando do interesse por política, verificamos que o modelo proposto confirma o resultado do teste de associação anterior uma vez que o tempo de envolvimento no OP produz efeito estatisticamente significativo em um nível bastante exigente (0.000) (Tabela 6). O coeficiente indica que cada ponto na escala de tempo produz elevação de 0.168 desvio-padrão na medida de interesse. $O$ coeficiente padronizado (Beta) revela que esse efeito perde 
em intensidade apenas para a escolaridade, sabidamente uma das mais importantes preditoras de uma série de variáveis relativas à cultura política (ALMOND e VERBA, 1989; MOISÉS, 1995; BORBA e RIBEIRO, 2010).

Tabela 6 - Preditores do Interesse por Política

\begin{tabular}{lccc}
\hline \multicolumn{1}{c}{ B } & Beta & Sig. \\
\hline Constante & 0.203 & & 0.193 \\
Tempo de OP & 0.168 & 0.184 & 0.000 \\
Sexo & 0.234 & 0.116 & 0.003 \\
Renda & 0.092 & 0.110 & 0.006 \\
Escolaridade & 0.234 & 0.425 & 0.000 \\
Idade & -0.001 & 0.011 & 0.775 \\
\hline \multicolumn{4}{c}{0.27} \\
\hline \multicolumn{4}{c}{ Fonte: NUPESAL (2000). }
\end{tabular}

Sobre a disposição para votar em uma situação de não obrigatoriedade, os resultados também continuam confirmando a relevância da experiência participativa (Tabela 7). O modelo logístico proposto revelou que apenas renda se mostrou um preditor relevante, além do tempo de OP. Enquanto cada elevação de faixa de renda familiar eleva em $54 \%$ a chance de um indivíduo pertencer ao grupo dos que votariam mesmo se isso não fosse obrigatório, elevações semelhantes na escala de tempo de envolvimento nessa inovação institucional aumentam essa chance em 39,3\%.

Tabela 7 - Preditores do Voto

\begin{tabular}{lcc}
\hline & Exp(B) & Sig. \\
\hline Constante & 0.204 & 0.000 \\
Tempo de OP & 1.393 & 0.004 \\
Sexo & 1.385 & 0.139 \\
Renda & 1.540 & 0.000 \\
Escolaridade & 1.003 & 0.654 \\
Idade & 1.111 & 0.287 \\
\hline \multicolumn{2}{c}{0.20} \\
\hline
\end{tabular}

Fonte: NUPESAL (2000).

Também se confirma o efeito da participação sobre a ocorrência de uma postura mais atenta às ações dos candidatos eleitos para os cargos públicos (Tabela 8). A chance de compor o grupo de entrevistados que costuma acompanhar as atividades dos eleitos é impactada apenas pelo tempo de OP em $41,1 \%$. O efeito de renda é um pouco maior, uma vez que cada elevação de faixa incrementa em $61,6 \%$ essa chance. Por fim, merece destaque o impacto de idade, pois como está mensurada em anos de vida apresenta efeito cumulativo considerável. O avançar de cada ano de vida eleva a probabilidade de acompanhamento em $47,5 \%$, o que revela um importante componente geracional nessa variável. 
Tabela 8 - Preditores do Acompanhamento dos Candidatos

\begin{tabular}{lcc}
\hline & $\operatorname{Exp}(\mathbf{B})$ & Sig. \\
\hline Constante & 0.124 & 0.000 \\
Tempo de OP & 1.411 & 0.010 \\
Sexo & 0.957 & 0.860 \\
Renda & 1.616 & 0.000 \\
Escolaridade & 1.009 & 0.242 \\
Idade & 1.475 & 0.001 \\
\hline & Pseudo R & 0.25 \\
\hline
\end{tabular}

Fonte: NUPESAL (2000).

Mais expressivo ainda foi o resultado do modelo tendo o Índice de Ativismo Eleitoral como variável dependente (Tabela 9). Além da nossa variável principal, apenas a escolaridade se mostrou importante preditora. Ainda assim, a comparação dos coeficientes padronizados indica efeito muito mais expressivo do tempo de OP (0.429 e 0.205, respectivamente). Considerando o coeficiente não padronizado, cada elevação na escala de tempo produz impacto de 1.329 desvio-padrão sobre o referido índice.

Tabela 9 - Preditores do Ativismo Eleitoral

\begin{tabular}{lccc}
\hline & \multicolumn{1}{l}{ B } & Beta & Sig. \\
\hline Constante & 1.346 & & 0.016 \\
Tempo de OP & 1.329 & 0.429 & 0.000 \\
Sexo & 0.078 & 0.011 & 0.780 \\
Renda & 0.113 & 0.039 & 0.352 \\
Escolaridade & 0.392 & 0.205 & 0.000 \\
Idade & -.002 & -0.007 & 0.856 \\
\hline \multicolumn{4}{c}{0.24} \\
\hline & $\mathrm{R}^{2}$ &
\end{tabular}

Resultado semelhante foi verificado também em relação à participação em associações, última variável dependente por nós analisada. Desta vez, entretanto, apenas a experiência no OP aparece como preditora, com efeito estatisticamente significativo (0.000). A intensidade desse impacto é também considerável, pois cada elevação na escala de tempo aumenta em 53,1\% a chance do indivíduo fazer parte do grupo dos que participam de grupos ou associações. 
Tabela 10 - Preditoras da Participação em Associações

\begin{tabular}{lcc}
\hline & $\operatorname{Exp}(\mathbf{B})$ & Sig. \\
\hline Constante & 0.158 & 0.000 \\
Tempo de OP & 1.531 & 0.000 \\
Sexo & 1.266 & 0.287 \\
Renda & 0.993 & 0.912 \\
Escolaridade & 1.007 & 0.322 \\
Idade & 0.991 & 0.926 \\
\hline
\end{tabular}

Fonte: NUPESAL (2000).

\section{Considerações Finais}

Como já destacado, o OP vem se constituindo em uma importante inovação institucional da democracia brasileira. Mais de uma centena de municípios já implantaram esse modelo de definição do orçamento público no Brasil (RIBEIRO e GRAZIA, 2003). Os defensores de tal modelo justificam sua adoção por diferentes motivos: racionalizar o gasto público, universalizar procedimentos, produzir accountability, produzir cidadania, promover educação política democrática, desenvolver o espírito e engajamento cívico, etc.

Veja-se que entre esses motivos, os relacionados à dimensão política, do civismo, são os que mais se destacam. Apesar disso, foram poucos os autores que trataram do assunto através de pesquisa empírica. Partindo desse diagnóstico, buscamos analisar a relação entre OP e algumas variáveis ligadas aos valores e atitudes políticas dos envolvidos nessa experiência participativa. Para isso, buscamos operacionalizar o estudo através de uma série de testes, que permitiram chegar às seguintes constatações:

a) Em primeiro lugar, o tempo de OP foi o único preditor que exerceu efeitos sobre todas as variáveis, o que atesta inequivocamente para o efeito socializador da experiência;

b) Nesse sentido, nosso estudo reafirma as conclusões de Fedozzi (2002) quanto ao OP se constituir num espaço de socialização política secundária, podendo atenuar os efeitos deletérios das desigualdades sociais (em especial da renda e escolaridade) sobre a incorporação de hábitos e atitudes democráticas pelos cidadãos;

c) Os efeitos produzidos pelo tempo de OP, porém se mostraram diferenciados, conforme a natureza das variáveis em análise. Ele parece impactar mais fortemente as predisposições para o engajamento individual em outras modalidades (associações, ativismo eleitoral), do que nos mecanismos de acompanhamento e controle dos governantes (accountability vertical), onde seu efeito foi menor que renda e idade. Tal constatação tem sido corroborada por outros trabalhos, como o Hartmann (2011), ao estudar o tema do aprendizado político dos participantes do OP do município de Concórdia/SC. 
d) Considerando que os efeitos mais significativos do OP são sobre as variáveis de ativismo, tem-se aí a possibilidade de outros tipos de leitura para tal experimento, relacionada a um possível efeito cooptação das elites partidárias e governamentais sobre os cidadãos que são atraídos para esses espaços de participação. Estudos como os de D'Avilla et al (2004) e Avritzer (2003) já alertaram a esse respeito e acreditamos que seja um fenômeno que deva ser investigado. Os dados empíricos que dispomos, porém não nos permitem ir além dessa constatação.

Por fim, cabe destacar que todos os testes realizados acima padecem de um limite metodológico que relativiza muitas de nossas conclusões. Nos referimos à natureza da direção da relação causal. Com os testes realizados, não temos condições de afirmar que os padrões observados devem-se, no caso específico, à participação no OP. A relação pode se dar no sentido inverso, ou seja, é possível que os indivíduos que participam do OP sejam aqueles que já possuíam maior confiança nas instituições, informação política, eram associados, etc. Essa hipótese continua plausível, mesmo considerando que nossa variável independente media o tempo de experiência no OP, ou seja, disposições políticas mais interessadas podem impactar inclusive o período de permanência nessa instituição participativa.

Uma das maneiras de resolver esse problema, como já mencionamos anteriormente, seria através da realização de pesquisas do tipo painel, onde se pode observar o comportamento dos atores em diferentes períodos. Como não dispúnhamos desse tipo de dado, salientamos para os limites de nossa análise.

Ednaldo Aparecido Ribeiro é Doutor e Mestre em Sociologia (UFPR), Professor do Departamento de Ciências Sociais da Universidade Estadual de Maringá.

E-mail: ednaldorip@uol.com.br

Julian Borba é Doutor em Ciência Política (UFRGS) e Mestre em Sociologia Política (UFSC), Professor do Departamento de Sociologia e Política da Universidade Federal de Santa Catarina.

E-mail: borbajulian@yahoo.com.br

\section{Referências:}

ABERS, Rebeca. Do clientelismo à cooperação: governos locais, políticas participativas e organização da sociedade civil em Porto Alegre. Cadernos do IPPUR, Rio de Janeiro, v. XII, n. 1, p. 37-42, jan.-jul. 1998.

ALMOND, Gabriel; POWELL JR; G. Bingham. Uma Teoria da Política Comparada. Rio de Janeiro: Zahar, 1972. 
ALMOND, Gabriel; VERBA, Sidney. The civic culture: political attitudes and democracy in five nations. Princeton: Princeton University Press, 1989.

ALVAREZ, Sônia A.; DAGNINO, Evelina; ESCOBAR, Arturo (Orgs.). Cultura e política nos movimentos sociais latino-americanos: novas leituras. Belo Horizonte: Ed. UFMG, 2000.

AVRITZER, Leonardo. Cultura política, atores sociais e democratização: uma crítica das teorias da transição para a democracia. Revista Brasileira de Ciências Sociais, São Paulo, v. 10, n. 28, p. 101-122, jun. 1995.

AVRITZER, Leonardo; NAVARRO, Zander (Orgs.). A inovação democrática no Brasil. São Paulo: Cortez, 2003.

AVRITZER, Leonardo; WAMPLER, Brian. The expansion of Participatory Budgeting in Brazil: an analysis of the successful cases based upon design and socio-economic indicators, 2008.2 Disponível em:<http://www.pbh.gov.br/redebrasileiraop/html/biblioteca/Relatorio_Banco _Mundial_CEnsoOP.pdf>. Acesso em: $13 \mathrm{fev} .2011$.

BAQUERO, Marcello. O papel dos adolescentes no processo de construção democrática no Brasil. Um estudo preliminar de socialização política. Porto Alegre: Editora da UFRGS, 1997. (Cadernos de Ciência Política, 8).

- Democracia, cultura e comportamento político: uma análise da situação brasileira. In: PERISSINOTTO, Renato M.; FUKS, Mário (Orgs.). Democracia: teoria e prática. Curitiba: Relume Dumará/Fundação Araucária, 2002. p. $105-139$.

Construindo uma outra sociedade: o capital social na estruturação da política participativa no Brasil. Revista de Sociologia e Política, Curitiba, n. 21, p. 83-108, nov. 2003.

et al. Bases de um novo contrato social? Impostos e Orçamento Participativo em Porto Alegre. Opinião Pública, Campinas, v. 11, n. 1, p. 94127, mar. 2005.

BORBA, Julian; LÜCHMANN, Lígia Helena H. (Orgs.). Orçamento Participativo: análise das experiências desenvolvidas em Santa Catarina. Florianópolis: Insular, 2007.

CARVALHO, José Murilo de. O motivo endêmico no imaginário social brasileiro. In: PANDOLFI, Dulce Chaves et al (Orgs.). Cidadania, justiça e violência. Rio de Janeiro: Fundação Getúlio Vargas, 1999. p. 19-43. 
CHAUÍ, Marilena. Brasil: mito fundador e sociedade autoritária. São Paulo: Editora Fundação Perseu Abramo, 2000.

DAHL, Robert. Poliarquia. São Paulo: Edusp, 1997.

D'AVILA, Paulo; LOMBARDO, Vladimyr; COELHO, Ana F. Acesso ao Poder, Clientelismo e Democracia: desconstruindo uma dicotomia. Revista Civitas Revista de Ciências Sociais, Porto Alegre, v. 4, n. 2, p. 211-233, jul.-dez. 2004.

FEDOZZI, Luciano. Orçamento participativo: reflexões sobre a experiência de Porto Alegre. Porto Alegre/Rio de Janeiro: Tomo editorial/Observatório de Políticas Urbanas e Gestão Municipal (FASE/IPPUR), 1997.

. O "eu e os outros": a construção da consciência social dos participantes do Orçamento Participativo de Porto Alegre. 2002. 339 f. Tese (Doutorado em Sociologia) - Programa de Pós-Graduação em Sociologia, Universidade Federal do Rio Grande do Sul, [2002].

HARTMANN, Fábio. Aprendizado político e democrático no Orçamento Participativo do município de Concórdia. 2011. 165 f. Dissertação (Mestrado em Sociologia Política) - Centro de Filosofia e Ciências Humanas, Universidade Federal de Santa Catarina [2011].

HUNTINGTON, Samuel. The third wave: democratization in the late twentieth century. Norman: University of Oklahoma, 1991.

INGLEHART, Ronald. The renaissance of political culture. American Political Science Review, Washington, v. 82, n. 4, p. 1203-1229, dec. 1988.

. Modernización y postmodernización: El cambio cultural, económico y político en 43 sociedades. Madrid: CIS/Siglo XXI, 2001.

INGLEHART, Ronald; WELZEL, Christian. Modernization, cultural change, and democracy: the human development sequence. New York: Cambridge University Press, 2005.

- Modernização, mudança cultural e democracia: a seqüência do desenvolvimento humano. São Paulo: Francis, 2009.

KRISCHKE, Paulo J. Cultura política: convergências e diferenças em Porto Alegre e Curitiba. Revista de Ciências Humanas, Florianópolis, n. 35, p. 142175, abr. 2004. 
A cultura política em Porto Alegre e Curitiba: democracia, modernização e o conteúdo da razão pública. Sociedade e Estado, Brasília, v. 20, n. 1, p. 39-71, jan.-abr. 2005.

LÜCHMANN, Lígia Helena. Possibilidades e limites da democracia deliberativa: a experiência do Orçamento Participativo de Porto Alegre. 2002. 215 f. Tese (Doutorado em Ciências Sociais) - Instituto de Filosofia e Ciências Humanas, UNICAMP [2002].

Democracia participativa e aprendizado político: lições da experiência brasileira. Florianópolis, 2008 (mimeo). Disponível em: <http://www.npms.ufsc.br>. Acesso em: 10 fev. 2011.

LÜCHMANN, Lígia Helena H.; BORBA, Julian. O orçamento participativo como modelo alternativo de gestão pública: uma análise das experiências brasileiras. Florianópolis, 2010 (mimeo).

MONCLAIRE, Stéphane. Democracia, transição e consolidação: precisões sobre conceitos. Revista de Sociologia e Política, Curitiba, n. 17, p. 61-74, nov. 2001.

MULLER, Edward N.; SELIGSON, Mitchell A. Civic culture and democracy: The question of causal relationships. American Political Science Review, Washington, v. 88, n. 3, p. 635-652, sep. 1994.

NORRIS, Pippa. Democratic Phoenix: political activism worldwide. Cambridge: Cambridge University Press, 2002.

NUPESAL. Núcleo de Pesquisas sobre a América Latina. Comportamento político e eleições. Porto Alegre: UFRGS, 2000. [Base de Dados].

O'DONNEL, Guillermo. Hiatos, continuidades e perspectivas democráticas. In: REIS, Fábio Wanderley; O'DONNELL, Guillermo (Orgs.). A democracia no Brasil, dilema e perspectiva. São Paulo: Vértice/Revista dos Tribunais, 1988. p. 72-86.

PONTUAL, Pedro de Carvalho. O processo educativo no Orçamento Participativo: aprendizados dos atores da sociedade civil e do estado. 2001. 281 f. Tese (Doutorado em Educação) - Faculdade de Educação, Pontifícia Universidade Católica de São Paulo, [2001].

POWERS, Daniel A.; XIE, Yu. Statistical methods for categorical data analysis. London: Emeral, 2008. 
PUTNAM, Robert. Comunidade e democracia: a experiência da Itália moderna. Rio de Janeiro: Ed. FGV, 1997.

RENNÓ, Lúcio. Teoria da Cultura Política: vícios e virtudes. BIB, Rio de Janeiro, n. 45, p. 71-91, jan.-jun. 1998.

RIBEIRO, Ana Clara T.; GRAZIA, Grazia de. Experiências de Orçamento Participativo no Brasil: período de 1997-2000. Petrópolis: Vozes, 2003.

SCHMIDT, João Pedro. Equilíbrio de baixa intensidade: capital social e socialização política dos jovens brasileiros na virada do século. In: BAQUERO, Marcello (Org.). Reinventando a sociedade na América latina: cultura política, gênero, exclusão e capital social. Porto Alegre: UFRGS/CNDM, 2001. p. 119149.

VIGEVANI, Tullo; OLIVEIRA, Marcelo Fernandes de. América Latina: vulnerabilidade social e instabilidade democrática. In: DUPAS, Gilberto (Coord.). América Latina no início do século XXI. Rio de Janeiro/São Paulo: Fundação Konrad Adenauer/Ed. Unesp, 2005. p. 19-253.

VITULO, Gabriel E. Transitologia, consolidologia e democracia na América Latina: uma revisão crítica. Revista de Sociologia e Política, Curitiba, n. 17, p. 53-60, nov. 2001.

Texto recebido em 14/04/2011.

Aprovado em 25/05/2011. 